\title{
The combination of PLLA/PLGA/PCL composite scaffolds integrated with BMP-2-loaded microspheres and low-intensity pulsed ultrasound alleviates steroid-induced osteonecrosis of the femoral head
}

\author{
HANXIAO ZHU, ZHONGLI SHI, XUNZI CAI, XIAOBO YANG and CHENHE ZHOU \\ Department of Orthopaedic Surgery, The Second Affiliated Hospital of Zhejiang \\ University School of Medicine, Hangzhou, Zhejiang 310009, P.R. China
}

Received February 19, 2019; Accepted November 14, 2019

DOI: $10.3892 / \mathrm{etm} .2020 .9254$

\begin{abstract}
Low-intensity pulsed ultrasound (LIPUS), which has been previously reported to promote bone repair, is proposed to be a noninvasive form of therapy for the treatment of osteonecrosis. Bone fillers made from composite scaffolds have been demonstrated to be effective for preventing bone defects such as osteonecrosis. The present study aimed to investigate whether the application of LIPUS combined with bone morphogenetic protein-2 (BMP-2)-loaded poly-L-lactic acid/polylactic-co-glycolic acid/poly- $\varepsilon$-caprolactone (PLLA/PLGA/PCL) composite scaffolds can improve recovery in a rat model of steroid-induced osteonecrosis of the femoral head (ONFH). BMP-2-loaded PLGA microspheres incorporated into PLLA/PLGA/PCL composite scaffolds were constructed. Bilateral femoral head LIPUS intervention was conducted in rats with steroid-induced ONFH. LIPUS intervention alone contributed to the alleviation of osteonecrosis, in addition to improving load-carrying capacity and accelerated bone formation, angiogenesis and differentiation. Subsequently, femoral head parameters and assessment of load-carrying capacity, bone formation-related factors, and angiogenesis- and differentiation-related factors were measured in rats with or without implanted BMP-2-loaded PLLA/PLGA/PCL composite scaffolds. LIPUS combined with the implantation of PLLA/PLGA/PCL composite scaffolds loaded with BMP-2 microspheres protected rats against steroid-induced ONFH and improved load-carrying capacity,
\end{abstract}

Correspondence to: Dr Chenhe Zhou, Department of Orthopaedic Surgery, The Second Affiliated Hospital of Zhejiang University School of Medicine, 88 Jiefang Road, Hangzhou, Zhejiang 310009, P.R. China

E-mail: zhouchenhe@zju.edu.cn

Key words: steroid-induced osteonecrosis, osteonecrosis of femoral head, bone morphogenetic protein 2, polylactic-co-glycolic acid microspheres, poly-L-lactic acid/polylactic-co-glycolic acid/poly- $\varepsilon$ caprolactone composite scaffold bone formation, angiogenesis and differentiation. Together, these data support the use of BMP-2-loaded PLLA/PLGA/PCL composite scaffolds combined with LIPUS for ONFH as a potential alternative curative solution for treating bone diseases.

\section{Introduction}

Osteonecrosis of the femoral head (ONFH) is characterized by the collapse of the femoral head and by joint damage, and is caused by osteocyte death as a result of insufficient blood supply (1). ONFH is a destructive long-term condition with a poorly understood pathogenic mechanism $(2,3)$. A number of factors have been demonstrated to increase the risk of osteonecrotic lesions, including corticosteroid use, alcohol consumption, trauma and abnormalities in coagulation (4). In particular, the risk of steroid-induced osteonecrosis is dependent on drug dosage, the route of drug delivery and underlying disease states (5). At present, total hip replacement is the only invasive therapy for advanced osteonecrosis (6). Although low-intensity pulsed ultrasound (LIPUS) has been proposed as an alternative noninvasive therapeutic option for ONFH (7), it remains necessary to explore other effective strategies to minimize treatment time. The use of bone morphogenetic proteins (BMPs) and LIPUS have been studied intensively as a prospective augmentation approach for tendon-to-bone healing, where the delivery of BMPs into the bone defect area combined with LIPUS has garnered attention for its potential to prevent steroid-induced ONFH (8).

Biomaterials, including polymer scaffolds, possess physical, mechanical and chemical properties that can be designed to carry integrin signals, growth factors and cytokines for bone regeneration and repair (9). Bioactive small molecules can be encapsulated or entrapped into scaffolds in such a way that the final constructs are able release bioactive compounds into the area of interest in a controlled manner (10). Of note, the BMP family of proteins, including BMP-2, BMP-4 and BMP-7, have the potential to induce endochondral bone formation when implanted into mammals (11). BMP-2 has been previously demonstrated to be an osteoconductive growth factor beneficial for ONFH by promoting cartilage repair and inducing osteoblast proliferation or differentiation (12). Recombinant 
human BMP-2 (rhBMP-2) may improve the clinical efficacy of impacted bone graft surgery by enhancing bone repair in ONFH (13). Several types of biodegradable poly- $\alpha$-hydroxy acid polymers, including polylactic acid (PLA), polyglycolic acid (PGA) and their copolymer polylactic-co-glycolic acid (PLGA), are popular options for the construction of scaffolds in delivering rhBMP (14). rhBMP-2 tethered to biomaterials has been revealed to enhance the stability and retention of biomaterials in the location of regeneration (15). In recent decades, poly-L-lactic acid (PLLA), and poly- $\varepsilon$-caprolactone (PCL) have emerged as potentially viable biodegradable polymers for therapeutic tissue engineering $(16,17)$. Therefore, the present study aimed to investigate the ability of a novel composite PLLA/PLGA/PCL scaffold for BMP-2 delivery and to assess the therapeutic action of this system in conjunction with LIPUS in terms of bone formation, angiogenesis and differentiation in a rat model of steroid-induced ONFH.

\section{Materials and methods}

Ethics statement. All animal experiments were performed in accordance with the principles and procedures of the Guide for the Care and Use of Laboratory Animals (18). And approved by the Animal Ethics Committee of The Second Affiliated Hospital of Zhejiang University School of Medicine.

Encapsulation of BMP-2-loaded PLGA microspheres. PLGA microspheres were manufactured by double emulsion. Briefly, $0.25 \mathrm{~g}$ polyvinyl alcohol (PVA) hydrogel was diluted in $250 \mathrm{ml}$ deionized water in a beaker. The diluted PVA was subsequently stirred at $60^{\circ} \mathrm{C}$ until the PVA was dissolved and cooled to room temperature. PLGA $(0.3 \mathrm{~g})$ was dissolved in $3 \mathrm{ml}$ dichloromethane. The configured PVA and PLGA solutions were cooled separately in an ice bath. The PLGA solution was then mixed with $500 \mu \mathrm{l}$ PBS or PBS supplemented with BMP-2 (Recombinant Human Bone Morphogenetic Protein 2, $1 \mu \mathrm{g} / \mathrm{ml}$, Beijing BioLab Technology Co., Ltd.) or PBS supplemented with BSA ( $1 \mu \mathrm{g} / \mathrm{ml}$, Beijing BioLab Technology Co., Ltd.), ultrasonically dispersed using a sonicator $\left(4^{\circ} \mathrm{C}, 200 \mathrm{~W}\right.$, $20 \mathrm{kHz}, 10 \mathrm{sec}$ ) until the solution turned milky white and added dropwise into the PVA solution. The PVA-PLGA mixture was then ultrasonically dispersed until it turned milky white $\left(4^{\circ} \mathrm{C}\right.$, $200 \mathrm{~W}, 20 \mathrm{kHz}, 2 \mathrm{~min}$ ), following which the mixture was stirred using a magnetic stirrer for 4-5 $\mathrm{h}$ at room temperature to allow the evaporation of dichloromethane. After the microspheres were formed, the mixture was centrifuged at $1,776 \mathrm{xg}$ for $5 \mathrm{~min}$ at room temperature, where the resulting supernatant was discarded, the pellet was washed three times using deionized water. The pellet was then freeze-dried to obtain empty or BMP-2 loaded PLGA microspheres.

Determination of loading and encapsulation efficiency. In total, $10 \mathrm{mg}$ BMP-2-loaded microspheres were mixed with $0.9 \mathrm{ml}$ of $\mathrm{NaOH}$ solution $(1 \mathrm{M})$ and $0.1 \mathrm{ml}$ PBS. After the mixture was stirred at room temperature for $2 \mathrm{~h}, 1 \mathrm{ml} 0.9 \mathrm{M}$ $\mathrm{HCl}$ was added to neutralize the system. Protein concentration was then determined in $20 \mu \mathrm{l}$ of this sample using a bicinchoninic acid protein assay kit (BCA; Beyotime Institute of Biotechnology). BMP-2 protein content was then calculated in $2 \mathrm{ml}$ sample solution, which was equated as the mass of
BMP-2 in the microspheres. The loading and encapsulation efficiency of BMP-2 were calculated according to the following formulas: Loading efficiency $(\%)=($ mass of BMP-2 protein in microspheres)/(total mass of microspheres) $\mathrm{x} 100 \%$; encapsulation efficiency $(\%)=($ mass of BMP-2 protein in microspheres)/(mass of BMP-2 protein used in the initial batch) $\mathrm{x} 100 \%$.

Packaging of BMP-2-loaded PLGA microspheres into the scaffold. The mixture of PLLA, PLGA and PCL were dissolved in tetrahydrofuran at a mass ratio of 3:4:3 and stirred at $60^{\circ} \mathrm{C}$ until fully dissolved. The solution was then transferred to a $2.5 \mathrm{ml}$ syringe barrel and stored at $-80^{\circ} \mathrm{C}$ overnight. The frozen sample was then cut into $\sim 1 \mathrm{~mm}$ slices, placed in an ice-water mixture and incubated at $4^{\circ} \mathrm{C}$ for 3 days. Deionized water was renewed three times every day to completely displace the tetrahydrofuran. After three days of incubation, the PLLA/PLGA/PCL composite scaffold was removed and freeze-dried.

To obtain BMP-2-loaded microspheres packaged with the PLGA-PLLA/PLGA/PCL composite scaffold, a total of $10 \mathrm{mg}$ nanostructured BMP-2-loaded microspheres were first uniformly dissolved in $1 \mathrm{ml} \mathrm{n}$-hexane, where $500 \mu 1$ BMP-2-loaded microspheres/n-hexane mixture was dropped onto one side of the dried PLLA/PLGA/PCL nanofiber scaffold. After the n-hexane was volatilized at room temperature, the other side was packed with another $500 \mu 1$ BMP-2-loaded microspheres. Both sides of the BMP-2 microsphere-containing scaffold were immersed in a n-hexane-tetrahydrofuran mixture (9:1) to physically bind the microspheres to the PLLA/PLGA/PCL nano-scaffold, which was subsequently removed by vacuum drying in a dry box at $30^{\circ} \mathrm{C}$ for three days.

Scanning electron microscopy (SEM). The microspheres were first dissolved in ethanol after they were made, where an ultrasonic wave was applied in the ethanol solution containing the microspheres for dispersion purposes. The dispersed liquid was aspirated using a pipette gun and dropped onto the aluminum foil. After the anhydrous ethanol evaporated at room temperature, the aluminum foil was pasted onto the electron microscope carrier platform using a carbon conductive tape (Hitachi, Ltd.). For the scaffold samples, the conductive adhesive was directly pasted onto the carrier platform. The samples were then sprayed with gold sputter coating and observed by scanning electron microscopy (SEM) under an accelerating voltage $(10 \mathrm{kV})$ at x1,000 magnification. Using Image pro plus 6.0 software (Media cybernetics, Inc.) to measure the particle size of PLGA microspheres in SEM photos, the average particle size was estimated.

In vitro release of $B S A$ from the PLGA-PLLA/PLGA/PCL composite scaffold. This assay was performed in accordance with a previously described protocol (19). In brief, the BSA-PLGA-PLLA/PLGA/PCL composite scaffold containing 10 mg BSA ( $1 \mu \mathrm{g} / \mathrm{ml}$, Beijing BioLab Technology Co., Ltd.) microspheres was added into a $5 \mathrm{ml}$ centrifuge tube containing $2 \mathrm{ml}$ PBS (pH 7.4). The centrifuge tube was then placed on a shaking table at $37^{\circ} \mathrm{C}$ and $1,118 \mathrm{x}$ g. Upon reaching the configured time points (days 1-7, 14, 15, 17, 21, 22, 28, 30, 40, 
50 and 60), $1 \mathrm{ml}$ solution was replaced with $1 \mathrm{ml}$ fresh PBS The concentration of BSA released into the obtained solution was determined at each time point using a BCA kit.

Experimental rat model of ONFH. In total, 40 male Sprague-Dawley rats (age, 10 weeks; weight, 300-320 g; license number, SCXK 2009-0004; Hunan SJA Laboratory Animal Co., Ltd.) were raised for 1 week at the Laboratory Animal Center, The Second Affiliated hospital of Zhejiang University School of Medicine (Zhejiang, China) according to standard feeding conditions (temperature, $18-26^{\circ} \mathrm{C}$; humidity, $40-70 \%$; light-dark cycle, 12:12 h; access to water and food, ad libitum) with 5 rats per cage. The rats were randomly divided into four groups $(n=10)$ : Normal group (sham-operated rats); Model group (rats with osteonecrosis); LIPUS group (rats treated with LIPUS); and BMP-2 + LIPUS group (rats implanted with the BMP-2-loaded PLGA-PLLA/PLGA/PCL composite scaffold treated with LIPUS). All rats were provided with a standardized diet and were allowed unrestricted activities.

ONFH establishment. The osteonecrosis model was established in a total of 30 randomly selected rats (all rats apart from rats in the Normal group). In brief, the rats were intraperitoneally injected with lipopolysaccharide $(20 \mu \mathrm{g} / \mathrm{kg})$ on day 0 and 1, once per day at an interval of $24 \mathrm{~h}$. Furthermore, both gluteal muscles were alternately injected with methylprednisolone $(20 \mathrm{mg} / \mathrm{kg})$ on days 2,3 and 4 once a day at an interval of $24 \mathrm{~h}$. At present, controversy remains regarding the use of hormones alone or in combination with adjuvants, including methylprednisolone and endotoxin (20). To ensure successful establishment of the model, methylprednisolone was administered in conjunction with lipopolysaccharide $(20 \mu \mathrm{g} / \mathrm{kg})$ to induce hormone-related osteonecrosis (21). In sham-operated rats, the abdominal cavity and gluteal muscles were injected with a $0.9 \%$ sodium chloride solution (normal saline) at the same time points as the model rats as aforementioned.

LIPUS and BMP-2 intervention. Rats in the LIPUS and the BMP-2 + LIPUS groups were subjected to bilateral femoral head LIPUS intervention (30 mW/cmz; Cosmogamma ${ }^{\circledR} \mathrm{US} 13$; AC International) two weeks after model establishment, for 20 min per day for 12 weeks until sacrifice. For the rats in the BMP-2 + LIPUS group, the scaffolds were inserted into the rats $24 \mathrm{~h}$ prior to LIPUS treatment. The rats were first anesthetized with pentobarbital sodium $(40 \mathrm{mg} / \mathrm{kg})$. The femoral head area was first sterilized, where an incision was made to expose the femoral head. A hole $3 \mathrm{~mm}$ in diameter was then made on the posteromedial surface of the femoral neck using a drill. The composite scaffold was made into a cylinder with a diameter of $\sim 3 \mathrm{~mm}$ and a thickness of $3 \mathrm{~mm}$, which were then implanted into the defect of rats prior to suture. Before irradiation, all rats were anaesthetized by an intraperitoneal injection of pentobarbital sodium $(40 \mathrm{mg} / \mathrm{kg})$. In detail, the hip joint was located to accurately determine the irradiation points by shaking the bilateral hind thighs. Following fur removal, the probe was smeared with a coupling agent to ensure close skin adherence. The dietary, mental and physical changes of all rats were monitored weekly. Healthy mental status was defined as actively inquisitive but without biting, mental depression, drowsiness or laziness. The fasting body weights of all the rats were measured using an electronic scale before modeling every week. The rats were treated for 12 weeks. Throughout the study, if any rats were adjudged to be suffering in accordance with the items of 'endpoints in animal study proposals' in the 'animal care and use procedures' section of the National Institutes of Health guidelines (22), they would be immediately sacrificed by an intraperitoneal injection of pentobarbital $(150 \mathrm{mg} / \mathrm{kg})$.

Micro-CT scan. All rats were euthanized by an intraperitoneal injection of pentobarbital $(150 \mathrm{mg} / \mathrm{kg}) 12$ weeks after ONFH establishment. The femoral head of the rats was extracted and fixed with $10 \%$ paraformaldehyde at $4^{\circ} \mathrm{C}$ for $4 \mathrm{~h}$ before the bone architecture was observed using a micro-CT scanner (scanning resolution, $45 \mu \mathrm{m}$; GE eXplore 120 model; GE Healthcare). The central region of the femoral head was set as the region of interest. The parameters measured were as follows: Bone mineral density (BMD), bone volume/total volume (BV/TV), trabecular number (Tb.N), trabecular thickness (Tb.Th) and trabecular separation (Tb.Sp).

Monitoring of biomechanical variables. The proximal femoral specimen was mounted on an experimental platform of a universal mechanical tester (ZwickRoell GmBH). The femur was kept moist during the experiment. A steel needle was placed directly above the femoral head and horizontal to the proximal femoral force line. A three-point bending test was performed on a three-point bending device to determine the maximum bending load and displacement of the femoral head by applying the following parameters: Maximum loading weight, $20 \mathrm{~kg}$; span at both ends, $20 \mathrm{~m}$; and loading speed, $5 \mathrm{~mm} / \mathrm{min}$.

Measurement of calcium $(\mathrm{Ca})$ and phosphorus $(P)$ content. The biomechanically measured femur was dried at $95^{\circ} \mathrm{C}$ and ground into a powder. A total of $0.1 \mathrm{~g}$ accurately weighed femur powder was placed in a digestion tube, soaked in $8 \mathrm{ml}$ nitric acid overnight at room temperature and detached according to the following procedure: Stage I at 1,600 W, heated for $5 \mathrm{~min}$ and maintained at $120^{\circ} \mathrm{C}$ for $2 \mathrm{~min}$; and stage II at $1,600 \mathrm{~W}$, heated for $8 \mathrm{~min}$ and maintained at $185^{\circ} \mathrm{C}$ for $25 \mathrm{~min}$. The decomposition solution obtained was then brought to $50 \mathrm{ml}$ and diluted 50 times in water. The $\mathrm{Ca}$ and $\mathrm{P}$ contents in the femur were determined (MARS 5 Digestion Microwave System; CEM Microwave Technology, Ltd.) under the following operating conditions: Radio-frequency power, 1,150 W; auxiliary gas flow rate, $0.5 \mathrm{l} / \mathrm{min}$; flushing pumping rate, $50 \mathrm{r} / \mathrm{min}$; analysis pumping rate, $50 \mathrm{r} / \mathrm{min}$; stable pumping time, $5 \mathrm{sec}$; and maximum integration time of the signal, $30 \mathrm{sec}$.

Angiogenesis assay. The femoral head tissues were fixed with $10 \%$ formaldehyde at $4{ }^{\circ} \mathrm{C}$ overnight and decalcified with $10 \%$ EDTA at room temperature until the tissue becomes soft. Paraffin-embedded tissues were then sliced into $5-\mu \mathrm{m}$ thick sections, which was then incubated at $60^{\circ} \mathrm{C}$ overnight. Prior to dewaxing, the tissue sections were placed at room temperature for $60 \mathrm{~min}$, following which they were rehydrated in xylene for $20 \mathrm{~min}$ and then a descending gradient of anhydrous ethanol (95\% ethanol and 70\% ethanol, $5 \mathrm{~min}$ 
respectively). Endogenous catalase was then blocked using $3 \% \mathrm{H}_{2} \mathrm{O}_{2}$ for $15 \mathrm{~min}$ at room temperature. This is followed by the antigen retrieval step, in which the tissue sections were incubated in $0.1 \mathrm{M}$ citric acid solution with $\mathrm{pH} 6.0$, at $100^{\circ} \mathrm{C}$ for $20 \mathrm{~min}$. The tissue sections were then blocked using $10 \%$ goat serum (cat. no. ab138478; Abcam) at $37^{\circ} \mathrm{C}$ for $30 \mathrm{~min}$, following which they were incubated with anti- $\alpha$-SMA antibody (1:100; cat. no. BM0002; Wuhan Boster Biological Technology, Ltd.), overnight at $4^{\circ} \mathrm{C}$. The next day, the sections were incubated with anti $\operatorname{IgG}$ secondary antibody (1:500; cat. no. G-21040; Thermo Fisher Scientific, Inc.) at $37^{\circ} \mathrm{C}$ for $30 \mathrm{~min}$, following which they were treated with reagents in the DAB Kit according to manufacturer's protocols (Wuhan Boster Biological Technology, Ltd.). The tissues sections were then stained with hematoxylin for $30 \mathrm{sec}$ at room temperature and dehydrated using an ascending alcohol gradient followed by xylene. After mounting the sections using neutral resin, staining was then observed under a light microscope (magnification, x200, Olympus CX41; Olympus Corporation). Five sections were randomly selected for the assessment of vessel number and diameter.

Reverse transcription-quantitative polymerase chain reaction $(R T-q P C R)$. Total RNA was extracted from tissues in accordance with the specifications of TRIzol ${ }^{\circledR}$ reagent (Thermo Fisher Scientific, Inc.) and $15 \mathrm{ng}$ the RNA was reverse transcribed into cDNA using PrimeScript ${ }^{\mathrm{TM}}$ RT reagent Kit with gDNA Eraser (Takara Biotechnology Co., Ltd.) according to manufacturer's protocol $\left(42^{\circ} \mathrm{C}\right.$ for $15 \mathrm{~min}$ and $85^{\circ} \mathrm{C}$ for $\left.5 \mathrm{sec}\right)$. qPCR was subsequently conducted in an ABI 7500 qPCR instrument using SYBR ${ }^{\circledR}$ Premix Ex Taq ${ }^{\mathrm{TM}}$ II (Takara Bio, Inc.). The thermocycling conditions were as follows: Initial denaturation at $95^{\circ} \mathrm{C} 1 \mathrm{~min}$; followed by 40 cycles of $95^{\circ} \mathrm{C}$ $10 \mathrm{sec}$ and $62^{\circ} \mathrm{C}$ for $25 \mathrm{sec}$. The primer sequences used for qPCR were synthesized by The Beijing Genomics Institute (Beijing, China) and are listed in Table I. The expression of target genes was calculated using the $2^{-\Delta \Delta \mathrm{Cq}}$ method (23) with GAPDH as the internal reference.

Western blot analysis. Total protein was extracted from the cell lysates or tissues using 1\% Triton X-100 solution, which was quantified using a BCA kit (Sigma-Aldrich; Merck $\mathrm{KGaA})$. The protein samples (50 $\mu \mathrm{g}$ per lane) were separated by $10 \%$ SDS-PAGE was transferred onto polyvinylidene fluoride membranes. The membrane was subsequently blocked with $50 \mathrm{~g} / 1 \mathrm{skim}$ milk powder at room temperature for $1 \mathrm{~h}$ and washed three times with Tris-buffered saline supplemented with $0.1 \%$ Tween-20 (TBS-T). Afterwards, the membrane was incubated overnight at $4{ }^{\circ} \mathrm{C}$ with the following primary rabbit anti-rat antibodies (all from Abcam): BMP-2 (1:1,000; cat. no. ab6285), transforming growth factor- $\beta 1$ (TGF- $\beta 1 ; 1: 100$; cat. no. ab64715), runt-related transcription factor 2 (RUNX2; 1:1,000; cat. no. ab76956), Collagen I (Col I; 1:3,000; cat. no. ab6308), Osteocalcin (OCN; 1:500; cat. no. ab93876) and $\beta$-actin (1:5,000; cat. no. ab8227). The membranes were then washed a further three times with TBS-T and incubated with a horseradish peroxidase-conjugated secondary antibody against Immunoglobulin G (1:3,000; ab205718; Abcam) for $1 \mathrm{~h}$ at room temperature, followed by further washing with TBS-T. Protein signals were detected using an enhanced chemiluminescence
Table I. Primers for reverse transcription quantitative polymerase chain reaction.

\begin{tabular}{ll}
\hline Gene & \multicolumn{1}{c}{ Primer sequence } \\
\hline BMP-2 & F: 5'-GGGACCCGCTGTCTTCTAGT-3' \\
& R: 5'-TCAACTCAAATTCGCTGAGGAC-3' \\
TGF- 31 & F: 5'-CCACCTGCAAGACCATCGAC-3' \\
& R: 5'-CTGGCGAGCCTTAGTTTGGAC-3' \\
RUNX2 & F: 5'-GCACAAACATGGCCAGATTCA-3' \\
& R: 5'-AAGCCATGGTGCCCGTTAG-3' \\
Col I & F: 5'-GACATGTTCAGCTTTGTGGACCTC-3' \\
& R: 5'-GGGACCCTTAGGCCATTGTGTA-3' \\
Osteocalcin & F: 5'-GCAATAAGGTAGTGAACAGACTCC-3' \\
& R: 5'-CCATAGATGCGTTTGTAGGCGG-3' \\
GAPDH & F: 5'-CATGAGAAGTATGACAACAGCCT-3' \\
& R: 5'-AGTCCTTCCACGATACCAAAGT-3'
\end{tabular}

$\mathrm{F}$, forward; R, reverse; BMP-2, bone morphogenetic protein 2; TGF- $\beta 1$, transforming growth factor $\beta 1$; RUNX2, runt-related transcription factor 2; Col I, type I collagen.

fluorescence detection kit (EMD Millipore) and quantified using Image Quant software (version 5.2, GE Healthcare Life Sciences).

Alizarin red staining. MC3T3-E1 cells (Type Culture Collection of the Chinese Academy of Sciences), usually cultured in 90\% $\alpha$-MEM media (cat. no. 11900024; Gibco; Thermo Fisher Scientific, Inc.) containing $1.5 \mathrm{~g} / 1 \mathrm{NaHCO}_{3}$, $43.2 \mathrm{mg} / 1$ inositol (Sinopharm Chemical Reagent Co., Ltd.), $8.82 \mathrm{mg} / \mathrm{l}$ folic acid (Sinopharm Chemical Reagent Co., Ltd.) and $7.8 \mathrm{mg} / 1 \beta$-mercaptoethanol (Sigma-Aldrich; Merck $\mathrm{KGaA}$ ) and $10 \% \mathrm{FBS}$ at $37^{\circ} \mathrm{C}$ and $5 \% \mathrm{CO}_{2}$. They were seeded into 12 -well plates at a density of $5 \times 10^{4}$ cells/well. Osteogenic induction medium (300 $\mu \mathrm{l}$; cat. no. PH-B-002; Puhe Biotechnology Co., Ltd.; https://puhe.biomart. $\mathrm{cn} /$ ) containing the PLLA/PLGA/PCL nano-scaffolds, PLGA-PLLA/PLGA/PCL composite scaffolds and BMP-2-PLGA-PLLA/PLGA/PCL composite scaffolds was then added to the cells, followed by incubation for 14 days at $37^{\circ} \mathrm{C}$. The medium was removed at the 14 th day. The cells were washed twice with PBS, fixed in $4 \%$ polyformaldehyde at $4^{\circ} \mathrm{C}$ for $4 \mathrm{~h}$, washed once with deionized water and stained with $2 \%$ alizarin red $(20 \mathrm{mg} / \mathrm{ml}, \mathrm{pH}=4.1-4.3)$ at room temperature for $20 \mathrm{~min}$. Any extra alizarin red was subsequently removed using deionized water followed by fluorescent microscopy (magnification: x200; Model: IX71; Olympus Corporation).

Statistical analysis. The experimental data were analyzed using SPSS 21.0 software (IBM Corp.). Measurement data are presented as the mean \pm standard deviation. Comparisons of two groups of data conforming to normal distribution were conducted using Student's t-test. Data between multiple groups were compared using one-way analysis of variance followed by Tukey's post hoc test. $\mathrm{P}<0.05$ was considered indicate a statistically significant difference. 

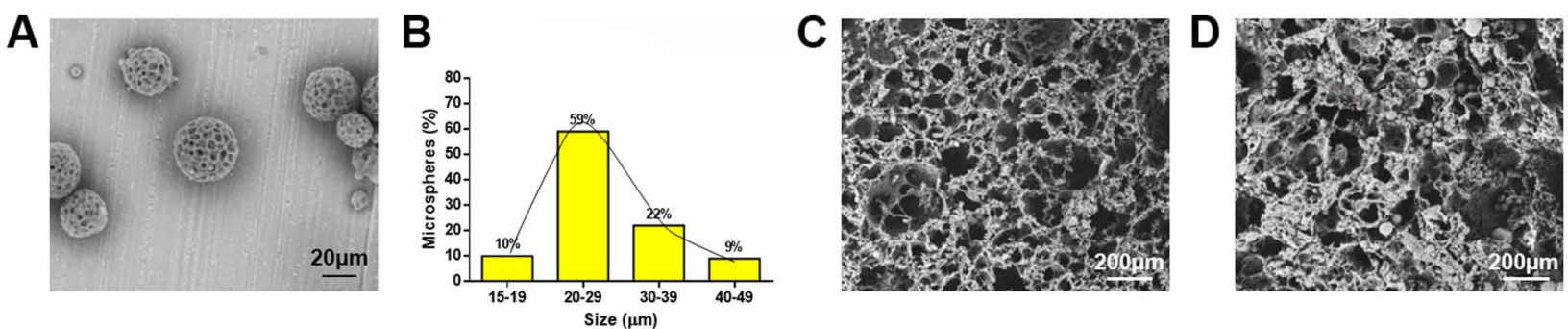

Figure 1. Identification of BMP-2-loaded PLGA microspheres into PLLA/PLGA/PCL composite scaffold. (A) Surface morphology of BMP-2-loaded PLGA microspheres observed under an electron microscope. (B) Particle size distribution of BMP-2-loaded PLGA nanoparticles. (C) Surface morphology of the PLLA/PLGA/PCL scaffold observed under an electron microscope. (D) Morphology of the PLGA-PLLA/PLGA/PCL composite scaffold loaded with BMP-2 observed under scanning electron microscope. BMP-2, bone morphogenetic protein-2; PLGA, polylactic-co-glycolic acid; PLLA, poly-L-lactic acid; PCL, poly- $\varepsilon$-caprolactone.

\section{Results}

Characterization of BMP-2-loaded PLGA microspheres incorporated into PLLA/PLGA/PCL composite scaffolds. The surface morphology of the BMP-2-loaded PLGA microspheres was observed using electron microscopy. The BMP-2-loaded PLGA microspheres exhibited relatively spherical shapes (Fig. 1A). The sizes of the particles were relatively uniform, with $81 \%$ of the PLGA microspheres presented with sizes between 20 and $39 \mu \mathrm{m}$ (Fig. 1B). According to the formula, the PLGA microspheres could load $0.77 \pm 0.62 \%$ of the BMP-2 protein with an encapsulation efficiency of $74.87 \pm 0.58 \%$, suggesting that the obtained microspheres could encapsulate more protein. However, the average protein loading rate was lower could be due to the large quantity of microspheres prepared. Under electron microscopic observation, the PLLA/PLGA/PCL scaffolds presented with a microscopically porous structure with good connectivity between the channels (Fig. 1C), which was suitable for cell adhesion and growth. Therefore, the PLLA/PLGA/PCL scaffold could be used as a biomimetic scaffold. After the BMP-2-loaded PLGA microspheres were incorporated into the PLLA/PLGA/PCL scaffold, it was observed that the PLGA microspheres were dispersed onto the surface and into some channels of the PLLA/PLGA/PCL scaffold structure (Fig. 1D).

PLLA/PLGA/PCL composite scaffold releases BSA in a sustained manner. The release of BSA from PLGA microspheres was rapid, as $>50 \%$ of BSA was already released within five days (Fig. 2). This sudden release of protein at the initial stage could be attributed to the small amount of protein adsorbed on the surface of microspheres. The release of BSA became slower from day 5 onwards, and $~ 90 \%$ of the BSA were released after 1 month. The reason for this observation was that the release of the BSA loaded inside the PLGA microspheres required a relatively long channel or a break in the matrix barrier. The results indicated that PLGA microspheres served as a microcarrier of protein.

Delivery of BMP-2 using the PLGA-PLLA/PLGA/ $P C L$ composite scaffold combined with LIPUS relieves steroid-induced femoral head necrosis. Following model construction, behavioral activities, diet, feces, mental status and body weights of the rats were observed regularly. The rats in the normal group exhibited good mental states, movement,

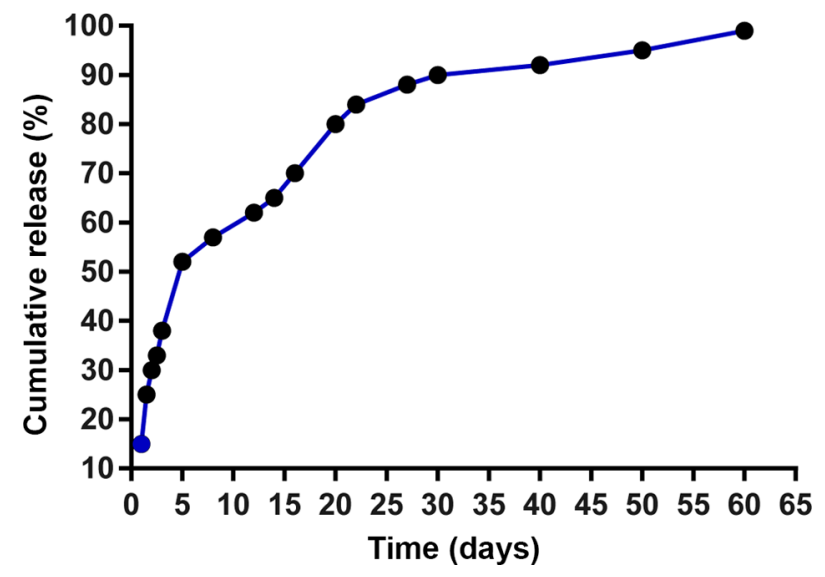

Figure 2. Cumulative release of bovine serum albumin from the PLGA-PLLA/PLGA/PCL composite scaffold in vitro. PLGA, polylactic-co-glycolic acid; PLLA, poly-L-lactic acid; PCL, poly- $\varepsilon$-caprolactone.

natural hair luster and no hair loss. Before LIPUS or BMP-2 treatment, the rats in the model, LIPUS and LIPUS + BMP-2 groups presented with thin and sloppy stool, mental depression, anorexia and emaciation after 3 days of model establishment. However, these symptoms disappeared after 2 weeks and the rats recovered. No rats were found dead or required immediate euthanasia during the experimental process. Micro-CT scan results revealed that the BMD, BV/TV, Tb.N and Tb.Th of the rats in the model group were significantly lower compared with those in the normal group $(\mathrm{P}<0.05)$, whereas the rats in the LIPUS and LIPUS + BMP-2 groups exhibited significantly higher BMD, BV/TV, Tb.N and Tb.Th compared with those in the model group $(\mathrm{P}<0.05)$. The Tb.Sp of rats in the model group was significantly higher compared with those in the normal group $(\mathrm{P}<0.05)$, whereas the Tb.Sp of rats in the LIPUS and LIPUS + BMP-2 groups were significantly lower compared with those in the model group $(\mathrm{P}<0.05$; Table II). These results indicated that LIPUS partially alleviated steroid-induced ONFH and that the use of BMP-2-loaded PLGA-PLLA/PLGA/PCL composite scaffolds combined with LIPUS was also conducive to the recovery of this disease.

Delivery of BMP-2 by PLGA-PLLA/PLGA/PCL composite scaffolds in addition to LIPUS improves load-carrying capacity. By measuring the kinesiological parameters, it was found that the maximum bending load and displacement of 
Table II. Micro-architectural parameters of femoral head in normal mice or mice with osteonecrosis.

\begin{tabular}{lcccc}
\hline Parameter & Normal & Model & LIPUS & LIPUS \pm BMP-2 \\
\hline BMD $(\mathrm{mg} / \mathrm{cc})$ & $648.94 \pm 39.25$ & $564.91 \pm 17.91^{\mathrm{a}}$ & $619.94 \pm 21.25^{\mathrm{b}}$ & $628.94 \pm 27.25^{\mathrm{b}}$ \\
BV/TV & $59.29 \pm 1.37$ & $37.93 \pm 1.04^{\mathrm{a}}$ & $49.29 \pm 1.13^{\mathrm{b}}$ & $53.29 \pm 1.18^{\mathrm{b}}$ \\
Tb.N $(1 / \mathrm{mm})$ & $2.95 \pm 0.14$ & $2.29 \pm 0.21^{\mathrm{a}}$ & $2.65 \pm 0.10^{\mathrm{b}}$ & $2.75 \pm 0.11^{\mathrm{b}}$ \\
Tb.Sp $(\mu \mathrm{m})$ & $174.36 \pm 53.30$ & $262.29 \pm 81.50^{\mathrm{a}}$ & $201.36 \pm 58.70^{\mathrm{b}}$ & $185.36 \pm 55.40^{\mathrm{b}}$ \\
Tb.Th $(\mu \mathrm{m})$ & $204.44 \pm 12.13$ & $158.32 \pm 9.23^{\mathrm{a}}$ & $174.44 \pm 10.47^{\mathrm{b}}$ & $187.44 \pm 11.83^{\mathrm{b}}$ \\
\hline
\end{tabular}

${ }^{a} \mathrm{P}<0.05$ vs. Normal and ${ }^{\mathrm{b}} \mathrm{P}<0.05$ vs. Model. BMD, bone mineral density; BV/TV, bone volume/total volume; Tb.N, trabecular number; Tb.Th, trabecular thickness; Tb.Sp, trabecular separation.

Table III. Kinematical parameters of femoral head in normal mice or mice with osteonecrosis.

\begin{tabular}{|c|c|c|c|c|}
\hline Parameter & Normal & Model & LIPUS & LIPUS + BMP-2 \\
\hline Maximum bending load (N) & $145.2 \pm 22.0$ & $124.6 \pm 8.0^{\mathrm{a}}$ & $134.1 \pm 13.7$ & $141.0 \pm 11.2^{\mathrm{b}}$ \\
\hline Maximum displacement $(\mathrm{cm})$ & $0.53 \pm 0.05$ & $0.42 \pm 0.04^{\mathrm{a}}$ & $0.46 \pm 0.04$ & $0.50 \pm 0.06^{\mathrm{b}}$ \\
\hline
\end{tabular}

${ }^{\mathrm{a}} \mathrm{P}<0.05$ vs. Normal and ${ }^{\mathrm{b}} \mathrm{P}<0.05$ vs. Model.

Table IV. Kinematical parameters of femoral head in normal mice or mice with osteonecrosis.

\begin{tabular}{lllll}
\hline Cotent & Normal & Model & LIPUS & LIPUS + BMP-2 \\
\hline Femoral Ca $(\mathrm{mg} / \mathrm{kg})$ & $261.1 \pm 10.3$ & $232.5 \pm 8.0^{\mathrm{a}}$ & $246.6 \pm 10.1$ & $253.7 \pm 7.7^{\mathrm{b}}$ \\
Femoral P $(\mathrm{mg} / \mathrm{kg})$ & $162.8 \pm 9.2$ & $134.4 \pm 7.5^{\mathrm{a}}$ & $148.4 \pm 8.7$ & $154.6 \pm 9.0^{\mathrm{b}}$ \\
\hline
\end{tabular}

${ }^{\mathrm{a}} \mathrm{P}<0.05$ vs. Normal and ${ }^{\mathrm{b}} \mathrm{P}<0.05$ vs. Model.

rats in the model group were significantly lower compared with those in the normal group $(\mathrm{P}<0.05)$. In contrast, the maximum bending load and displacement of the femoral head were significantly higher in the LIPUS + BMP-2 group compared with the model group $(\mathrm{P}<0.05$; Table III). Together, these data suggested that the load-carrying capacity of the femoral head of rats with steroid-induced ONFH was inferior compared with normal rats, which can be alleviated by LIPUS treatment and additively by the delivery of BMP-2 by PLGA-PLLA/PLGA/PCL composite scaffolds.

Delivery of BMP-2 by PLGA-PLLA/PLGA/PCL composite scaffold combined with LIPUS accelerates bone formation. During the bone formation process, calcium and phosphorus in the blood are deposited in bone tissues, in a process known as osteoid calcification, which is key to bone formation (24). By measuring $\mathrm{Ca}$ and $\mathrm{P}$ contents in rat femoral head samples it was found that those in the model group were significantly lower compared with the normal group $(\mathrm{P}<0.05)$. In comparison with the model group, the femoral $\mathrm{Ca}$ and $\mathrm{P}$ contents of were significantly higher in the LIPUS + BMP-2 group $(\mathrm{P}<0.05$; Table IV). These findings suggest that the implantation of BMP-2-loaded PLGA-PLLA/PLGA/PCL composite scaffold plus LIPUS treatment accelerated bone formation in rats with steroid-induced $\mathrm{ONFH}$.
Delivery of BMP-2 by PLGA-PLLA/PLGA/PCL composite scaffold in addition to LIPUS accelerates bone angiogenesis. Blood vessels were visualized in femoral head tissue sections stained with $\alpha$-SMA. The number and diameter of blood vessels were significantly reduced in the model group compared with the normal group $(\mathrm{P}<0.05)$, whereas the number of blood vessels was dramatically increased in the LIPUS + BMP-2 group compared with the model group ( $\mathrm{P}<0.05$; Fig. 3 ). The diameter of the blood vessels was slightly increased in the LIPUS + BMP-2 group compared with that in the model group but without statistical significance (Fig. 3C). These findings suggest that angiogenesis was promoted by the delivery of BMP-2 by PLGA-PLLA/PLGA/PCL composite scaffolds combined with LIPUS treatment.

Delivery of BMP-2 by PLGA-PLLA/PLGA/PCL composite scaffold in addition to LIPUS enhances osteocyte differentiation. The mRNA and protein expression of BMP-2, TGF- $\beta 1$, RUNX2, Col I and OCN in tissues were subsequently determined using RT-qPCR and western blot analysis. The expression of BMP- 2 , TGF- $\beta 1$, RUNX2, Col I and OCN was significantly lower in the model group compared that in the normal group $(\mathrm{P}<0.05)$, which was significantly reversed by either LIPUS alone or LIPUS + BMP-2 (P<0.05; Fig. 4A and B). The highest level of BMP-2 
A

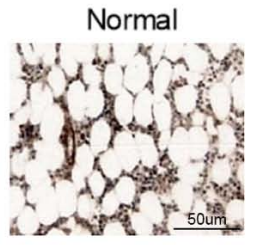

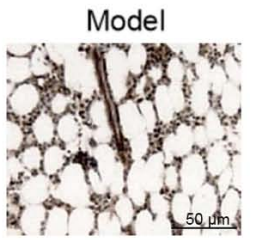

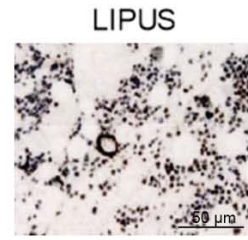

LIPUS+BMP-2

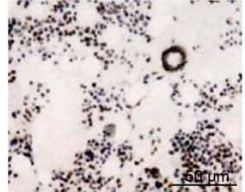

\section{B}
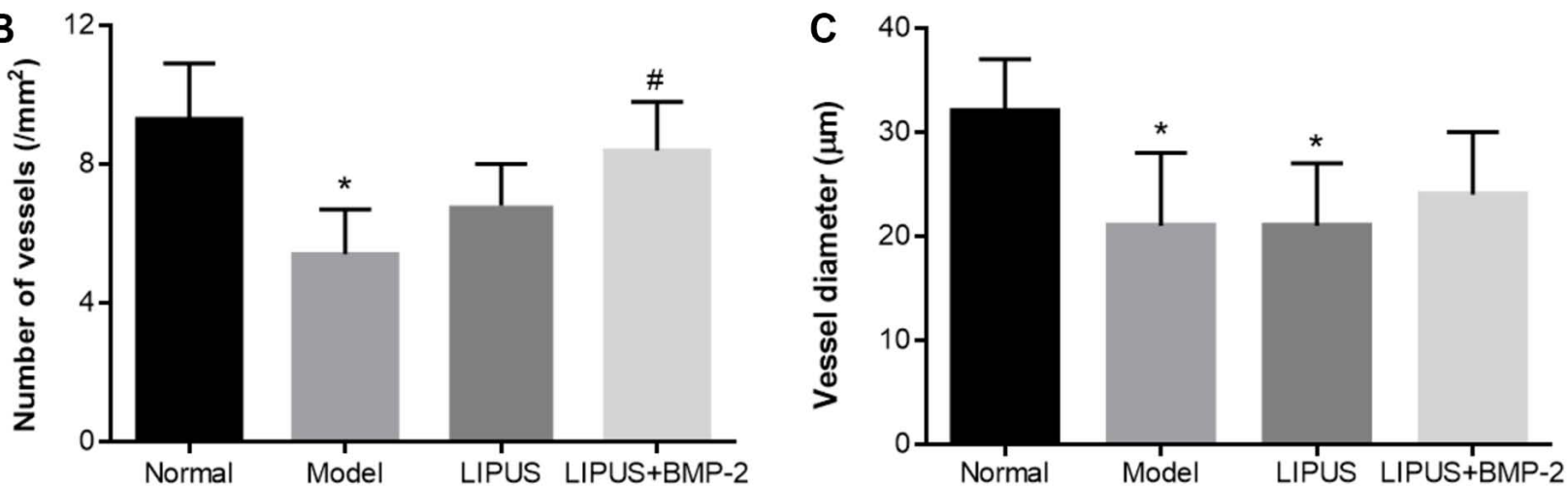

Figure 3. Delivery of BMP-2 by PLGA-PLLA/PLGA/PCL composite scaffold accelerates angiogenesis combined with LIPUS treatment. (A) Representative images displaying $\alpha$-SMA staining of femoral head sections collected from rats of the four groups. (B) Number of blood vessels as counted from images of femoral head sections. (C) Diameter of blood vessels as measured from images of femoral head sections. " $\mathrm{P}<0.05$ vs. Normal and ${ }^{\#} \mathrm{P}<0.05$ vs. Model. BMP-2, bone morphogenetic protein-2; PLGA, polylactic-co-glycolic acid; PLLA, poly-L-lactic acid; PCL, poly- $\varepsilon$-caprolactone; $\alpha$-SMA, $\alpha$-smooth muscle actin; LIPUS, low-intensity pulsed ultrasound; BMP-2, bone morphogenetic protein-2.

A

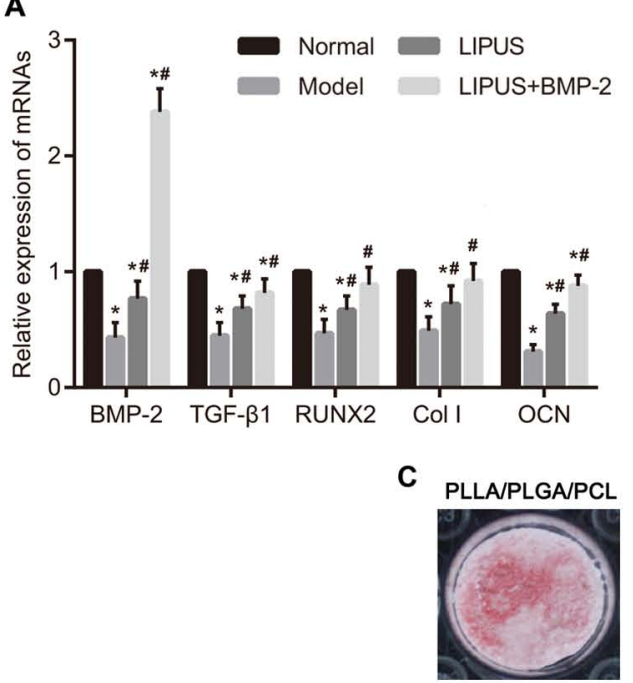

B
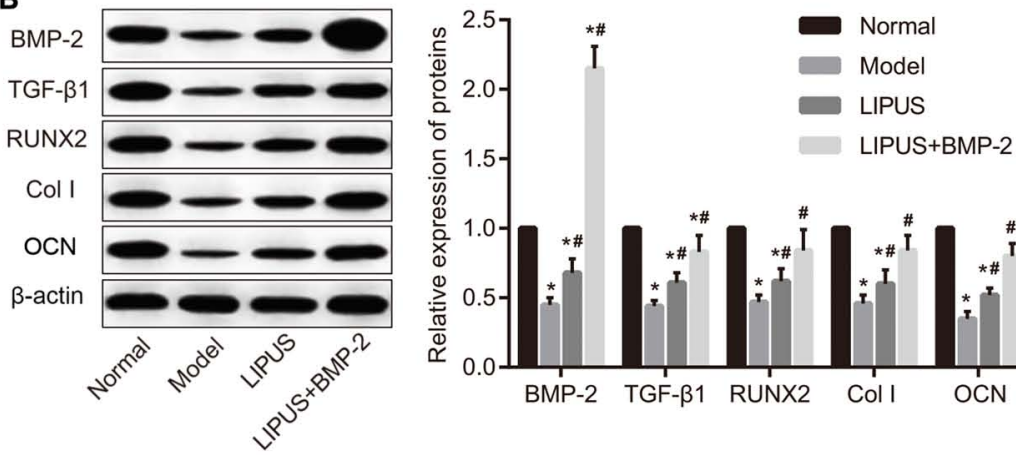

PLGA-PLLA/PLGA/PCL BMP-2-PLGA-PLLA/PLGA/PCL
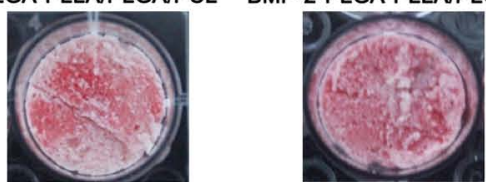

Figure 4. Delivery of BMP-2 by PLGA-PLLA/PLGA/PCL composite scaffold increases TGF- $\beta 1$, RUNX2, Col I and OCN expression combined with LIPUS treatment. (A) Measurement of BMP-2, TGF- $\beta 1$, RUNX2 and Col I and OCN mRNA expression by reverse transcription-quantitative PCR, performed using tissues. (B) Measurement of BMP-2, TGF- $\beta 1$, RUNX2, Col I and OCN protein expression by western blotting, performed using tissues. (C) Measurement of calcium deposition by alizarin red staining, performed using MC3T3-E1 cell lines. * $\mathrm{P}<0.05$ vs. Normal and "P<0.05 vs. Model. BMP-2, bone morphogenetic protein-2; PLGA, polylactic-co-glycolic acid; PLLA, poly-L-lactic acid; PCL, poly- $\varepsilon$-caprolactone; TGF- $\beta 1$, transforming growth factor $\beta 1$; RUNX2, runt-related transcription factor 2; Col I, type I collagen; OCN, osteocalcin.

was observed in the LIPUS + BMP-2 group, suggesting that BMP-2 was released following composite scaffold implantation, which subsequently induced bone formation. According to the results of alizarin red staining (Fig. 4C) in MC3T3-E1 cells, calcium deposition was markedly higher in the BMP-2-PLGA-PLLA/PLGA/PCL composite scaffolds group, compared with those treated with PLLA/PLGA/PCL nano-scaffolds and PLGA-PLLA/PLGA/PCL composite scaffolds. In conclusion, BMP-2 delivery through the PLGA-PLLA/PLGA/PCL composite scaffold in addition to
LIPUS treatment upregulated the expression of the osteoblast-specific transcription factors RUNX2, Col I and OCN.

\section{Discussion}

The sustained release of BMP-2 from nano-scaffolds is capable of enhancing bone regeneration, resulting in superior bone quality compared with the direct administration of BMP-2 (25). In the present study BMP-2-loaded PLGA microspheres were successfully incorporated into PLLA/PLGA/PCL composite 
scaffolds. The data suggested that BMP-2 delivered using PLLA/PLGA/PCL composite scaffolds promoted bone formation and repair in rat ONFH models in combination with LIPUS.

In the present study, it was determined that LIPUS alone or both LIPUS + BMP-2-loaded scaffolds increased BMD, BV/TV, Tb.N and Tb.Th but reduced Tb.Sp in rats with steroid-induced ONFH and improved bone load-carrying capacity. In addition, it was demonstrated that LIPUS partially alleviated steroid-induced femoral head necrosis, the effect of which was potentiated additively when combined with BMP-2 delivery using the PLGA-PLLA/PLGA/PCL composite scaffold. In a previous study, a porous PLGA/TCP composite scaffold incorporated with icaritin could repair bone defects and prevent hip joint collapse in a steroid-induced rabbit model of osteonecrosis (26), whilst 2.0\% nanosilver particle-PLGA composite grafts incorporated with BMP-2 contributed to the healing of femoral defects as a result of infection within 12 weeks with antibacterial activity in another study (27). Additionally, the implantation of PCL scaffolds loaded with BMP-2/BMP-7 nanocapsules accelerated the healing of iliac crest defects in rats (28), whereas BMP-2 conjugation into three-dimensional PCL scaffolds has been previously revealed to be a feasible approach in stimulating the osteoinductive capability of bone marrow stromal cells (29). PLLA nanosheets also had a good capacity for use as a sustained-release vehicle of rhBMP-2, enhancing bone regeneration (30). Considering the capability of PLGA, PCL and PLLA as nanocapsules in BMP-2 delivery, the present study first constructed a delivery system of BMP-2 using a PLLA/PLGA/PCL composite scaffold and provided evidence that the release of BMP-2 from the PLLA/PLGA/PCL composite scaffold potentiated protective effects against steroid-induced ONFH.

In the present study, LIPUS combined with the release of BMP-2 from the PLLA/PLGA/PCL composite scaffold was observed to increase $\mathrm{Ca}$ and $\mathrm{P}$ contents and upregulate the expression of the osteoblast-specific transcription factor RUNX2, Col I and OCN, further demonstrating its inductive role in bone formation and repair. LIPUS has been previously shown to enhance bone repair through osteogenesis and neovascularization in steroid-associated osteonecrosis (31). Mechanistically, LIPUS stimulates bone regeneration by exerting pro-osteogenic effects by promoting the expression of genes associated with osteogenesis in osteoblasts, including RUNX2, alkaline phosphatase and osteorix $(32,33)$. Col I stimulates the proliferation and osteogenesis of human mesenchymal stem cells (34). Increased expression of $\mathrm{OCN}$ is indicative of the therapeutic effects of deferoxamine on early-stage ONFH in a rabbit model (35), consistent with the present study. It has been previously demonstrated that daily LIPUS can stimulate the release of BMPs in the rat clonal cell line ROS 17/2.8 during osteogenic differentiation processes (36), whilst another study revealed that LIPUS enhanced rhBMP-2-induced bone formation (37). LIPUS induces angiogenesis through the upregulation of vascular endothelial growth factor (VEGF) expression, expediting osteoporotic fracture healing (38). Data from the present study suggest that blood vessels were more numerous and blood vessel diameters were greater following LIPUS treatment in combination with the implantation of BMP-2-containing PLGA-PLLA/PLGA/PCL composite scaffolds, implicating increased angiogenesis. As previously reported, LIPUS enhances angiogenesis by increasing VEGF after acute myocardial infarction (39). Additionally, BMP-2 treatment stimulated angiogenesis in human endothelial progenitor cells (40). The incorporation of either BMP-2 or VEGF inside porous scaffolds has been demonstrated to induce both angiogenesis and osteogenesis in a previous study (41). After osteonecrosis, the enlarged adipocytes caused blood vessel compression, resulting in reduced local blood supply and blood vessel diameter. Following LIPUS treatment and BMP-2 delivery, angiogenesis was induced. Since the newly formed blood vessels were small, the diameter of the blood vessels in this group was not markedly different from that of the blood vessels in the model group.

In the present study, TGF- $\beta$ signaling was induced following the implantation of the BMP-2-containing PLLA/PLGA/PCL composite scaffold plus LIPUS treatment. The TGF- $\beta$ signaling pathway has also been demonstrated to be an osteoclast-specific signaling pathway which can also induce bone formation (42). BMP and TGF- $\beta$ are two important factors associated with the proliferation and differentiation of osteoblasts (43), such that the interplay between TGF- $\beta$ and BMP signaling has been highlighted in osteoblast differentiation and bone formation $(44,45)$. Since TGF- $\beta$ has been shown to enhance BMP-9-stimulated osteogenesis of mesenchymal stem cells in a previous study (46), it can be speculated that TGF- $\beta$ signaling may participate in the mechanism underlying the protection of the BMP-2 delivered from PLLA/PLGA/PCL composite scaffolds against ONFH. However, further studies are necessary to test this hypothesis.

It should be noted that the number of samples in the present study was limited according to the Animal Ethics Committee of The Second Affiliated Hospital of Zhejiang University School of Medicine, suggesting the need for further research with a larger sample size. The effects of bare scaffolds on the alleviation of steroid-induced ONFH were acquiescently ruled out in the present study and should be seriously considered in future investigations.

Taken together, results in the present study demonstrate that the PLLA/PLGA/PCL composite scaffold is able to deliver BMP-2 into rats with steroid-induced ONFH, implicating PLLA/PLGA/PCL composite scaffold to be a potential carrier for BMP-2 delivery. In addition, the present study also demonstrated the combined application of BMP-2 and LIPUS contributed to bone formation and repair for steroid-induced ONFH. This strategy can be potentially applied for future clinical applications.

\section{Acknowledgements}

Not applicable.

\section{Funding}

The present study was funded by The Zhejiang Provincial Natural Science Foundation of China (grant no.LQ18H070002).

\section{Availability of data and materials}

The datasets used and/or analyzed during the current study are available from the corresponding author on reasonable request. 


\section{Authors' contributions}

HXZ and CHZ conceived, designed the current study, and developed the methodology. XZC, ZLS and XBY acquired and analyzed data. HXZ reviewed and revised the manuscript. The final version of the manuscript was read and approved by all authors.

\section{Ethical approval and consent to participate}

The present study was approved by The Second Affiliated Hospital, Zhejiang University School of Medicine (approval no. A2018014; Zhejiang, China).

\section{Patient consent for publication}

Not applicable.

\section{Competing interests}

The authors declare that they have no competing interests.

\section{References}

1. Tripathy SK, Goyal T and Sen RK: Management of femoral head osteonecrosis: Current concepts. Indian J Orthop 49: 28-45, 2015.

2. Issa K, Pivec R, Kapadia BH, Banerjee $S$ and Mont MA: Osteonecrosis of the femoral head: The total hip replacement solution. Bone Joint J 95-B (11 Suppl A): S46-S50, 2013.

3. Mont MA, Cherian JJ, Sierra RJ, Jones LC and Lieberman JR: Nontraumatic osteonecrosis of the femoral head: Where do we stand today? A ten-year update. J Bone Joint Surg Am 97: 1604-1627, 2015.

4. Zalavras CG and Lieberman JR: Osteonecrosis of the femoral head: Evaluation and treatment. J Am Acad Orthop Surg 22: 455-464, 2014.

5. Powell C, Chang C, Naguwa SM, Cheema G and Gershwin ME: Steroid induced osteonecrosis: An analysis of steroid dosing risk Autoimmun Rev 9: 721-743, 2010.

6. Powell C, Chang C and Gershwin ME: Current concepts on the pathogenesis and natural history of steroid-induced osteonecrosis. Clin Rev Allergy Immunol 41: 102-113, 2011.

7. Yan SG, Huang LY and Cai XZ: Low-intensity pulsed ultrasound: A potential non-invasive therapy for femoral head osteonecrosis. Med Hypotheses 76: 4-7, 2011.

8. Atesok K, Fu FH, Wolf MR, Ochi M, Jazrawi LM, Doral MN, Lubowitz JH and Rodeo SA: Augmentation of tendon-to-bone healing. J Bone Joint Surg Am 96: 513-521, 2014.

9. Agarwal R and Garcia AJ: Biomaterial strategies for engineering implants for enhanced osseointegration and bone repair. Adv Drug Deliv Rev 94: 53-62, 2015.

10. Balmayor ER: Targeted delivery as key for the success of small osteoinductive molecules. Adv Drug Deliv Rev 94 $13-27,2015$

11. Salazar VS, Gamer LW and Rosen V: BMP signalling in skeletal development, disease and repair. Nat Rev Endocrinol 12: 203-221, 2016.

12. Wang C, Zang H and Zhou D: Bone morphogenetic protein-2 exhibits therapeutic benefits for osteonecrosis of the femoral head through induction of cartilage and bone cells. Exp Ther Med 15: 4298-4308, 2018.

13. Sun W, Li Z, Gao F, Shi Z, Zhang Q and Guo W: Recombinant human bone morphogenetic protein-2 in debridement and impacted bone graft for the treatment of femoral head osteonecrosis. PLoS One 9: e100424, 2014.

14. Lo KW, Ulery BD, Ashe KM and Laurencin CT: Studies of bone morphogenetic protein-based surgical repair. Adv Drug Deliv Rev 64: 1277-1291, 2012.

15. King WJ and Krebsbach PH: Growth factor delivery: How surface interactions modulate release in vitro and in vivo. Adv Drug Deliv Rev 64: 1239-1256, 2012.
16. Fitzgerald R, Bass LM, Goldberg DJ, Graivier MH and Lorenc ZP: Physiochemical characteristics of poly-L-lactic acid (PLLA). Aesthet Surg J 38 (Suppl 1): S13-S17, 2018.

17. Martins AF, Facchi SP, da Camara PCF, Camargo SEA, Camargo CHR, Popat KC and Kipper MJ: Novel poly( $\varepsilon$-caprolactone)/amino-functionalized tannin electrospun membranes as scaffolds for tissue engineering. J Colloid Interface Sci 525: 21-30, 2018

18. Barthold SW, Bayne KA, Davis MA, Bayne K and Davis M: Guide for the care and use of laboratory animals. Publication no. 85-23(rev.) 327: 963-965, 2011.

19. Mercado AE, Ma J, He X and Jabbari E: Release characteristics and osteogenic activity of recombinant human bone morphogenetic protein-2 grafted to novel self-assembled poly(lactide-co-glycolide fumarate) nanoparticles. J Control Release 140: 148-156, 2009.

20. Drescher W, Bünger MH, Weigert K, Bünger C and Hansen ES: Methylprednisolone enhances contraction of porcine femoral head epiphyseal arteries. Clin Orthop Relat Res: 112-117, 2004.

21. Dong Y, Li Y, Huang C, Gao K and Weng X: Systemic application of teriparatide for steroid induced osteonecrosis in a rat model. BMC Musculoskelet Disord 16: 163, 2015.

22. Mcpherson C: Regulation of animal care and research? NIH's opinion. J Anim Sci 51: 492-496, 1980.

23. Livak KJ and Schmittgen TD: Analysis of relative gene expression data using real-time quantitative PCR and the 2(-Delta Delta C(T)) method. Methods 25: 402-408, 2001.

24. Florencio-Silva R, Sasso GR, Sasso-Cerri E, Simões MJ and Cerri PS: Biology of bone tissue: Structure, function, and factors that influence bone cells. Biomed Res Int 2015: 421746, 2015

25. Tian H, Du J, Wen J, Liu Y, Montgomery SR, Scott TP, Aghdasi B, Xiong C, Suzuki A, Hayashi T, et al: Growth-factor nanocapsules that enable tunable controlled release for bone regeneration. ACS Nano 10: 7362-7369, 2016.

26. Qin L, Yao D, Zheng L, Liu WC, Liu Z, Lei M, Huang L, Xie X, Wang X, Chen Y, et al: Phytomolecule icaritin incorporated PLGA/TCP scaffold for steroid-associated osteonecrosis: Proof-of-concept for prevention of hip joint collapse in bipedal emus and mechanistic study in quadrupedal rabbits. Biomaterials 59: 125-143, 2015.

27. Zheng Z, Yin W, Zara JN, Li W, Kwak J, Mamidi R, Lee M, Siu RK, Ngo R, Wang J, et al: The use of BMP-2 coupled-nanosilver-PLGA composite grafts to induce bone repair in grossly infected segmental defects. Biomaterials 31: 9293-9300, 2010.

28. Yilgor P, Yilmaz G, Onal MB, Solmaz I, Gundogdu S, Keskil S, Sousa RA, Reis RL, Hasirci N and Hasirci V: An in vivo study on the effect of scaffold geometry and growth factor release on the healing of bone defects. J Tissue Eng Regen Med 7: 687-696, 2013.

29. Zhang H, Migneco F, Lin CY and Hollister SJ: Chemicallyconjugated bone morphogenetic protein-2 on three-dimensional polycaprolactone scaffolds stimulates osteogenic activity in bone marrow stromal cells. Tissue Eng Part A 16: 3441-3448, 2010.

30. Huang KC, Yano F, Murahashi Y, Takano S, Kitaura Y, Chang SH, Soma K, Ueng SWN, Tanaka S, Ishihara K, et al: Sandwich-type PLLA-nanosheets loaded with BMP-2 induce bone regeneration in critical-sized mouse calvarial defects. Acta Biomater 59: 12-20, 2017.

31. Zhu H, Cai X, Lin T, Shi Z and Yan S: Low-intensity pulsed ultrasound enhances bone repair in a rabbit model of steroid-associated osteonecrosis. Clin Orthop Relat Res 473: 1830-1839, 2015.

32. Gleizal A, Ferreira S, Lavandier B, Simon B, Béziat JL and Béra JC: The impact of low intensity pulsed ultrasound on mouse skull bone osteoblast cultures. Rev Stomatol Chir Maxillofac 111: 280-285, 2010 (In French).

33. Zhou XY, Wu SY, Zhang ZC, Wang F, Yang YL, Li M and Wei XZ: Low-intensity pulsed ultrasound promotes endothelial cell-mediated osteogenesis in a conditioned medium coculture system with osteoblasts. Medicine (Baltimore) 96: e8397, 2017.

34. Tsai KS, Kao SY, Wang CY, Wang YJ, Wang JP and Hung SC: Type I collagen promotes proliferation and osteogenesis of human mesenchymal stem cells via activation of ERK and Akt pathways. J Biomed Mater Res A 94: 673-682, 2010.

35. Li J, Fan L, Yu Z, Dang X and Wang K: The effect of deferoxamine on angiogenesis and bone repair in steroid-induced osteonecrosis of rabbit femoral heads. Exp Biol Med (Maywood) 240: 273-280, 2015. 
36. Suzuki A, Takayama T, Suzuki N, Kojima T, Ota N, Asano S and Ito K: Daily low-intensity pulsed ultrasound stimulates production of bone morphogenetic protein in ROS 17/2.8 cells. J Oral Sci 51: 29-36, 2009.

37. Wijdicks CA, Virdi AS, Sena K, Sumner DR and Leven RM: Ultrasound enhances recombinant human BMP-2 induced ectopic bone formation in a rat model. Ultrasound Med Biol 35: 1629-1637, 2009.

38. Cheung WH, Chow SK, Sun MH, Qin L and Leung KS: Low-intensity pulsed ultrasound accelerated callus formation, angiogenesis and callus remodeling in osteoporotic fracture healing. Ultrasound Med Biol 37: 231-238, 2011.

39. Shindo T, Ito K, Ogata T, Hatanaka K, Kurosawa R, Eguchi K, Kagaya Y, Hanawa K, Aizawa K, Shiroto T, et al: Low-Intensity pulsed ultrasound enhances angiogenesis and ameliorates left ventricular dysfunction in a mouse model of acute myocardial infarction. Arterioscler Thromb Vasc Biol 36: 1220-1229, 2016.

40. Chen WC, Chung $\mathrm{CH}$, Lu YC, Wu MH, Chou PH, Yen JY, Lai YW, Wang GS, Liu SC, Cheng JK, et al: BMP-2 induces angiogenesis by provoking integrin $\alpha 6$ expression in human endothelial progenitor cells. Biochem Pharmacol 150: 256-266, 2018.

41. Lv J, Xiu P, Tan J, Jia Z, Cai H and Liu Z: Enhanced angiogenesis and osteogenesis in critical bone defects by the controlled release of BMP-2 and VEGF: Implantation of electron beam melting-fabricated porous Ti6Al4V scaffolds incorporating growth factor-doped fibrin glue. Biomed Mater 10: 035013, 2015.
42. Weivoda MM, Ruan M, Pederson L, Hachfeld C, Davey RA, Zajac JD, Westendorf JJ, Khosla S and Oursler MJ: Osteoclast TGF- $\beta$ receptor signaling induces Wnt1 secretion and couples bone resorption to bone formation. J Bone Miner Res 31: 76-85, 2016.

43. Fujiwara $\mathrm{M}$ and Ozono K: Cytokines and osteogenesis. Clin Calcium 24: 845-851, 2014 (In Japanese).

44. Liu DD, Zhang JC, Zhang Q, Wang SX and Yang MS: TGF- $\beta$ /BMP signaling pathway is involved in cerium-promoted osteogenic differentiation of mesenchymal stem cells. J Cell Biochem 114: 1105-1114, 2013.

45. Chen G, Deng C and Li YP: TGF- $\beta$ and BMP signaling in osteoblast differentiation and bone formation. Int J Biol Sci 8: 272-288, 2012.

46. Li XL, Liu YB, Ma EG, Shen WX, Li H and Zhang YN: Synergistic effect of BMP9 and TGF- $\beta$ in the proliferation and differentiation of osteoblasts. Genet Mol Res 14: 7605-7615, 2015.

c) (i) $\ominus$ This work is licensed under a Creative Commons Attribution-NonCommercial-NoDerivatives 4.0 International (CC BY-NC-ND 4.0) License. 